\title{
On the Explicit Four-Step Methods with Vanished Phase-Lag and its First Derivative
}

\author{
T. E. Simos ${ }^{1,2, *}$
}

${ }^{1}$ Department of Mathematics, College of Sciences, King Saud University, P. O. Box 2455, Riyadh 11451, Saudi Arabia

${ }^{2}$ Laboratory of Computational Sciences, Department of Informatics and Telecommunications, Faculty of Economy, Management and Informatics, University of Peloponnese, GR-221 00 Tripolis, Greece

\begin{abstract}
In the present paper, we will investigate a family of explicit four-step methods first introduced by Anastassi and Simos [1] for the case of vanishing of phase-lag and its first derivative. These methods are efficient for the numerical solution of the Schrödinger equation and related initial-value or boundary-value problems with periodic and/or oscillating solutions. As we mentioned before the main scope of this paper is the study of the elimination of the phase-lag and its first derivative of the family of the methods to be investigated. A comparative error and stability analysis will be presented for the studied family of four-step explicit methods. The new obtained methods will finally be tested on the resonance problem of the Schrödinger equation in order to examine their efficiency.
\end{abstract}

Keywords: Phase-lag, initial value problems, oscillating solution, symmetric, multistep, Schrödinger equation

\section{Introduction}

In this paper the numerical solution of special second-order initial-value problems of the form

$$
y^{\prime \prime}(x)=f(x, y), y\left(x_{0}\right)=y_{0} \text { and } y^{\prime}\left(x_{0}\right)=y_{0}^{\prime}
$$

with an periodical and/or oscillatory solutions are investigated. The main characteristic of the mathematical models of the above mentioned problems is that the ordinary differential equations which describe the above models are of second order in which the first derivative $y^{\prime}$ does not appear explicitly (see for numerical methods for these problems [3] - [32] and references therein).

\section{Analysis of the Phase-lag For Symmetric Multistep Methods}

For the numerical integration of the above mentioned initial value problem (1), one can use multistep methods of the form

$$
\sum_{i=0}^{k} c_{i} y_{n+i}=h^{2} \sum_{i=0}^{k} b_{i} f\left(x_{n+i}, y_{n+i}\right)
$$

with $k$ steps over the equally spaced intervals $\left\{x_{i}\right\}_{i=0}^{k} \in$ $[a, b]$ and $h=\left|x_{i+1}-x_{i}\right|, \quad i=0(1) k-1$, where $h$ is called stepsize of integration.

If the method is symmetric then $c_{i}=c_{k-i}$ and $b_{i}=b_{k-i}$, $i=0(1)\left\lfloor\frac{k}{2}\right\rfloor$.

The Multistep Method (2) is associated with the operator

$L(x)=\sum_{i=0}^{k} c_{i} u(x+i h)-h^{2} \sum_{i=0}^{k} b_{i} u^{\prime \prime}(x+i h)$

where $u \in C^{2}$.

Definition 1.[1] The multistep method (2) is called algebraic of order $q$ if the associated linear operator $L$ vanishes for any linear combination of the linearly independent functions $1, x, x^{2}, \ldots, x^{q+1}$.

Application of a symmetric $2 m$-step method, that is for $i=-m(1) m$, to the scalar test equation

$$
y^{\prime \prime}=-\phi^{2} y
$$

leads to the following difference equation:

$$
\begin{array}{r}
A_{m}(v) y_{n+m}+\ldots+A_{1}(v) y_{n+1}+A_{0}(v) y_{n} \\
+A_{1}(v) y_{n-1}+\ldots+A_{m}(v) y_{n-m}=0
\end{array}
$$

\footnotetext{
* Corresponding author e-mail: tsimos.conf@ gmail.com
} 
where $v=\phi h, h$ is the step length and $A_{j}(v) j=0(1) m$ are polynomials of $v$. by:

The associated with (5) characteristic equation is given

$$
\begin{array}{r}
A_{m}(v) \lambda^{m}+\ldots+A_{1}(v) \lambda+A_{0}(v) \\
+A_{1}(v) \lambda^{-1}+\ldots+A_{m}(v) \lambda^{-m}=0
\end{array}
$$

Lambert and Watson (1976) introduce the following definitions:

Definition 2.A symmetric 2 m-step method with characteristic equation given by (6) is said to have an interval of periodicity $\left(0, v_{0}^{2}\right)$ if, for all $v \in\left(0, v_{0}^{2}\right)$, the roots $\lambda_{i}, i=1(1) 2 m$ of Eq. (6) satisfy:

$$
\lambda_{1}=e^{i \theta(v)}, \lambda_{2}=e^{-i \theta(v)}, \text { and }\left|\lambda_{i}\right| \leq 1, i=3(1) 2 m
$$

where $\theta(v)$ is a real function of $v$.

Definition 3.[14], [15] For any method corresponding to the characteristic equation (6) the phase-lag is defined as the leading term in the expansion of

$$
t=v-\theta(v)
$$

Then if the quantity $t=O\left(v^{p+1}\right)$ as $v \rightarrow \infty$, the order of phase-lag is $p$.

Definition 4.[2] A method for which the phase-lag vanishes, is called phase-fitted

Theorem 1.[14] The symmetric 2m-step method with characteristic equation given by (6) has phase-lag order $p$ and phase-lag constant $c$ given by

$$
\begin{gathered}
-c v^{p+2}+O\left(v^{p+4}\right)= \\
\frac{2 A_{m}(v) \cos (m v)+\ldots+2 A_{j}(v) \cos (j v)+\ldots+A_{0}(v)}{2 m^{2} A_{m}(v)+\ldots+2 j^{2} A_{j}(v)+\ldots+2 A_{1}(v)}
\end{gathered}
$$

The formula proposed from the above theorem gives us a direct method to calculate the phase-lag of any symmetric $2 m$ - step method.

In our case, the symmetric four-step method has phase-lag order $p$ and phase-lag constant $c$ given by:

$$
\begin{gathered}
-c v^{p+2}+O\left(v^{p+4}\right)=\frac{T_{0}}{T_{1}} \\
T_{0}=2 A_{2}(v) \cos (2 v)+2 A_{1}(v) \cos (v)+A_{0}(v) \\
T_{1}=8 A_{2}(v)+2 A_{1}(v)
\end{gathered}
$$

\section{The Family of Explicit Four-Step Methods with Vanished Phase-Lag and Its First Derivative}

From the form (2) and without loss of generality we assume $c_{k}=1$ and we can write

$$
y_{n+k}+\sum_{i=0}^{k-1} c_{i} y_{n+i}=h^{2} \sum_{i=0}^{k} b_{i} f\left(x_{n+i}, y_{n+i}\right),
$$

If the method is symmetric then $c_{i}=c_{k-i}$ and $b_{i}=b_{k-i}, \quad i=0(1)\left\lfloor\frac{k}{2}\right\rfloor$.

From the form (11) with $k=4$ and $b_{4}=0$ we get the form of the explicit symmetric four-step methods [1]:

$$
\begin{array}{r}
y_{n+4}+c_{3}\left(y_{n+3}+y_{n+1}\right)+c_{2} y_{n+2}+y_{n}= \\
=h^{2}\left[b_{1}\left(f_{n+3}+f_{n+1}\right)+b_{0} f_{n+2}\right]
\end{array}
$$

where $f_{i}=y^{\prime \prime}\left(x_{i}, y_{i}\right), i=n-1(1) n+1$.

\subsection{First Case}

Considering (12), we choose:

$$
c_{0}=-2 c_{1}-2, c_{1}=-\frac{1}{10}
$$

The choice was based on the results of the investigation presented in [1]. Based on this study, the above values give for the method (12) the higher accuracy.

Demanding now the above method to have the phaselag and its first derivative vanished, the following system of equations is produced:

$$
\begin{aligned}
\frac{T_{2}}{\frac{39}{5}+2 v^{2} b_{1}} & =0 \\
-\frac{T_{3}}{\left(10 v^{2} b_{1}+39\right)^{2}} & =0
\end{aligned}
$$

where

$$
\begin{aligned}
T_{2} & =2 \cos (2 v)+2\left(-\frac{1}{10}+v^{2} b_{1}\right) \cos (v)-\frac{9}{5}+v^{2} b_{0} \\
T_{3} & =100 \sin (v) v^{4} b_{1}^{2}+400 \sin (v) \cos (v) v^{2} b_{1} \\
& +380 \sin (v) v^{2} b_{1}+400 v b_{1}(\cos (v))^{2} \\
& -800 v b_{1} \cos (v)+1560 \sin (v) \cos (v) \\
& -390 v b_{0}-380 v b_{1}-39 \sin (v)
\end{aligned}
$$

Solving the above system of equations, we obtain the coefficients of the new proposed method:

$$
b_{0}=\frac{1}{5} \frac{T_{4}}{\sin (v) v^{3}}, b_{1}=\frac{1}{10} \frac{T_{5}}{\sin (v) v^{3}}
$$

where

$$
\begin{aligned}
T_{4} & =24 \sin (v) v+5 v \sin (3 v)-\cos (2 v) \\
& -8 \cos (v)+10 \cos (3 v)-1 \\
T_{5} & =-20 v \sin (2 v)+18-20 \cos (2 v) \\
& +\sin (v) v+2 \cos (v)
\end{aligned}
$$


The following Taylor series expansions should be used in the cases that the formulae given by (15) are subject to heavy cancellations for some values of $|v|$ :

$$
\begin{aligned}
b_{0} & =\frac{5}{4}+\frac{161}{600} v^{2}-\frac{1541}{20160} v^{4} \\
& +\frac{473}{67200} v^{6}-\frac{28811}{79833600} v^{8} \\
& +\frac{3991}{342144000} v^{10}-\frac{1023167}{3487131648000} v^{12} \\
& +\frac{5317391}{1778437140480000} v^{14}-\frac{60114757}{243290200817664000} v^{16} \\
& -\frac{216374219}{12164510040883200000} v^{18}+\ldots \\
b_{1} & =\frac{53}{40}-\frac{161}{1200} v^{2}+\frac{943}{201600} v^{4} \\
& -\frac{13}{134400} v^{6}+\frac{109}{798336000} v^{8} \\
& -\frac{457}{4790016000} v^{10}-\frac{28033}{3170119680000} v^{12} \\
& -\frac{3202931}{3556874280960000} v^{14}-\frac{31704163}{347557429739520000} v^{16} \\
& -\frac{1574039107}{170303140572364800000} v^{18}+\ldots
\end{aligned}
$$

The behavior of the coefficients is given in the following Figure 1.

The new obtained method (12) (mentioned as FourStepI) with the coefficients given by (15)-(16) has a local truncation error which is given by:

$$
L T E_{\text {FourStepI }}=\frac{161 h^{6}}{2400}\left(y_{n}^{(6)}+2 \phi^{2} y_{n}^{(4)}+\phi^{4} y_{n}^{(2)}\right)+O\left(h^{8}\right)
$$

\subsection{Second Case}

Considering (12), we choose:

$$
c_{0}=-2 c_{1}-2, c_{1}=-\frac{9}{20}
$$

The choice was based on the results of the investigation presented in [1]. Based on this study, the above values give for the method (12) the medium accurate solution.

Requesting the above method to have the phase-lag and its first derivative equal to zero, the following system of equations is produced:

$$
\begin{aligned}
\frac{T_{6}}{\frac{71}{10}+2 v^{2} b_{1}} & =0 \\
-\frac{T_{7}}{\left(20 v^{2} b_{1}+71\right)^{2}} & =0
\end{aligned}
$$
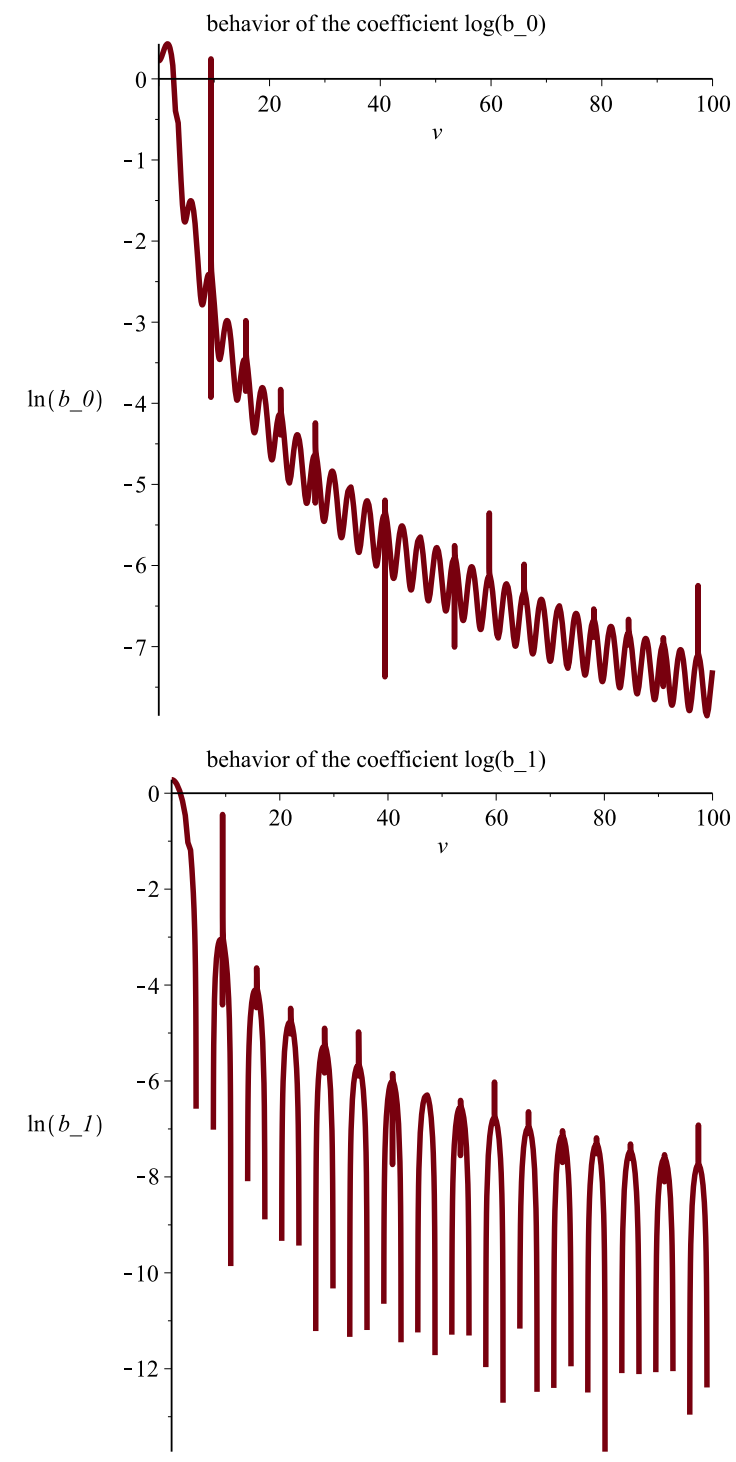

Fig. 1: Behavior of the coefficients of the new proposed method given by (15) for several values of $v=\phi h$.

where

$$
\begin{aligned}
T_{6} & =2 \cos (2 v)+2\left(-\frac{9}{20}+v^{2} b_{1}\right) \cos (v)-\frac{11}{10}+v^{2} b_{0} \\
T_{7} & =400 \sin (v) v^{4} b_{1}^{2}+1600 \sin (v) \cos (v) v^{2} b_{1} \\
& +1600 v b_{1}(\cos (v))^{2}+1240 \sin (v) v^{2} b_{1} \\
& -3200 v b_{1} \cos (v)+5680 \sin (v) \cos (v) \\
& -1420 v b_{0}-1240 v b_{1}-639 \sin (v)
\end{aligned}
$$


The coefficients of the new proposed method are obtained as solution of the above system of equations :

$$
b_{0}=\frac{1}{10} \frac{T_{8}}{\sin (v) v^{3}}, b_{1}=1 / 20 \frac{T_{9}}{\sin (v) v^{3}}
$$

where

$$
\begin{aligned}
T_{8} & =41 \sin (v) v+10 v \sin (3 v)-9 \cos (2 v) \\
& -2 \cos (v)+20 \cos (3 v)-9 \\
T_{9} & =9 \sin (v) v-40 v \sin (2 v) \\
& -40 \cos (2 v)+18 \cos (v)+22
\end{aligned}
$$

The following Taylor series expansions should be used in the cases that the formulae given by (20) are subject to heavy cancellations for some values of $|v|$ :

$$
\begin{aligned}
b_{0} & =\frac{23}{24}+\frac{329}{1200} v^{2}-\frac{1039}{13440} v^{4} \\
& +\frac{25339}{3628800} v^{6}-\frac{11719}{31933440} v^{8} \\
& +\frac{331}{29952000} v^{10}-\frac{7440421}{20922789888000} v^{12} \\
& -\frac{11781241}{3556874280960000} v^{14}-\frac{11049547}{12476420554752000} v^{16} \\
& -\frac{6020361833}{72987060245299200000} v^{18}+\ldots \\
b_{1} & =\frac{311}{240}-\frac{329}{2400} v^{2}+\frac{589}{134400} v^{4} \\
& -\frac{919}{7257600} v^{6}-\frac{4619}{1596672000} v^{8} \\
& -\frac{16703}{41513472000} v^{10}-\frac{8357161}{209227898880000} v^{12} \\
& -\frac{28821899}{7113748561920000} v^{14}-\frac{4529189}{11033569198080000} v^{16} \\
& -\frac{42499050289}{1021818843434188800000} v^{18}+\ldots
\end{aligned}
$$

The behavior of the coefficients is given in the following Figure 1.

The new obtained method (12) (mentioned as FourStepII) with the coefficients given by (20)-(21) has a local truncation error which is given by:

$L T E_{\text {FourStepII }}=\frac{329 h^{6}}{4800}\left(y_{n}^{(6)}+2 \phi^{2} y_{n}^{(4)}+\phi^{4} y_{n}^{(2)}\right)+O\left(h^{8}\right)$

\section{Comparative Error Analysis}

We will study the following methods (Case I) ${ }^{1}$ :

1 The results for the Case II is analogous
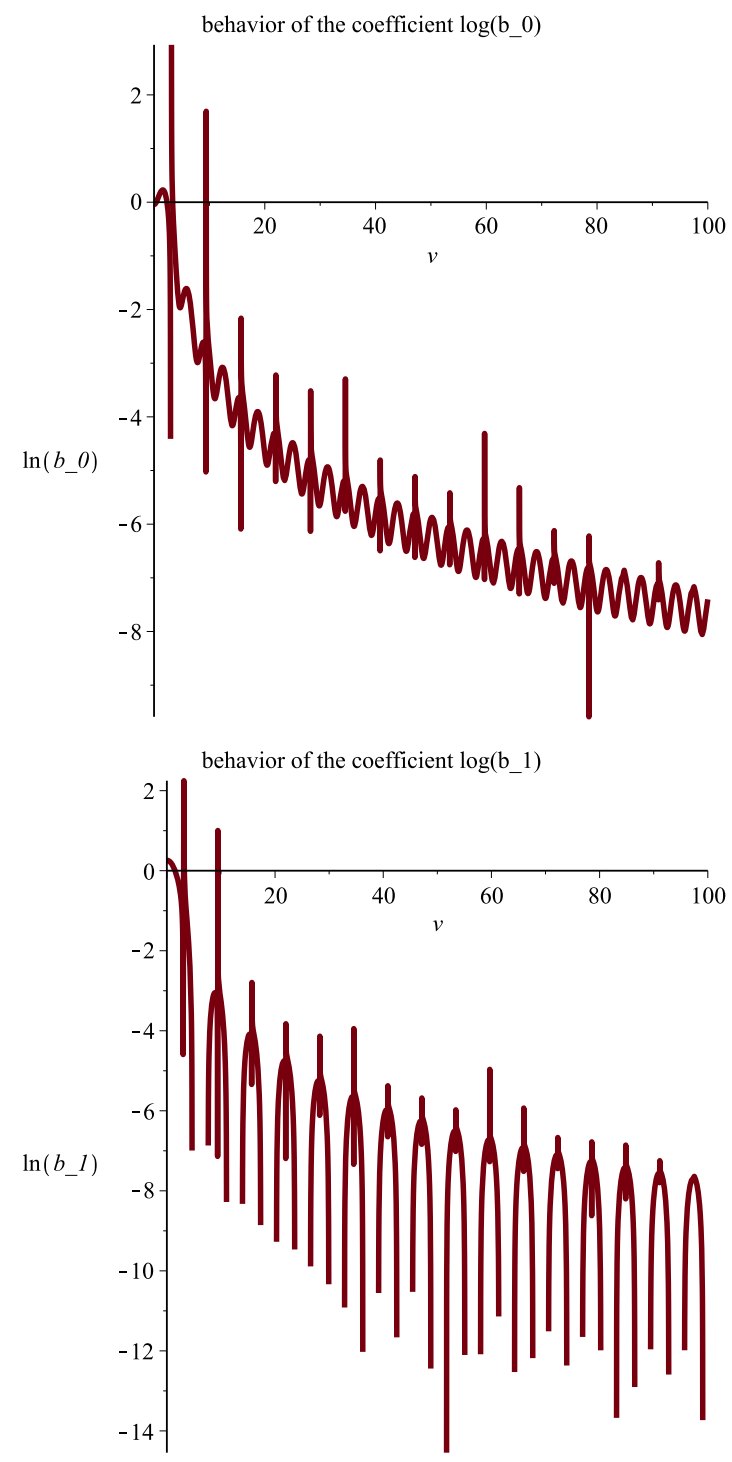

Fig. 2: Behavior of the coefficients of the new proposed method given by (20) for several values of $v=\phi h$.

4.1 Classical Method(i.e. the method (12) with constant coefficients of the Case I)

$$
L T E_{C L}=\frac{161 h^{6}}{2400} y_{n}^{(6)}+O\left(h^{8}\right)
$$

\subsection{The Method with Vanished Phase-Lag Produced in [1]}

$$
L T E_{\text {MethAnasSim }}=\frac{161 h^{6}}{2400}\left(y_{n}^{(6)}+\phi^{2} y_{n}^{(4)}\right)+O\left(h^{8}\right)
$$




\subsection{The New Proposed Method with Vanished}

Phase-Lag and its First Derivative Produced in Section 3

$L T E_{\text {FourStepII }}=\frac{161 h^{6}}{2400}\left(y_{n}^{(6)}+2 \phi^{2} y_{n}^{(4)}+\phi^{4} y_{n}^{(2)}\right)+O\left(h^{8}\right)$

The procedure contains the following stages

-Computation of the derivatives which presented in the formulae of the Local Truncation Errors. These computations lead to the following formulae:

$$
\begin{aligned}
& y_{n}^{(2)}=\left(V(x)-V_{c}+G\right) y(x) \\
& y_{n}^{(3)}=\left(\frac{d}{d x} g(x)\right) y(x)+(g(x)+G) \frac{d}{d x} y(x) \\
& y_{n}^{(4)}=\left(\frac{d^{2}}{d x^{2}} g(x)\right) y(x)+2\left(\frac{d}{d x} g(x)\right) \frac{d}{d x} y(x) \\
& +(g(x)+G)^{2} y(x) \\
& y_{n}^{(5)}=\left(\frac{d^{3}}{d x^{3}} g(x)\right) y(x)+3\left(\frac{d^{2}}{d x^{2}} g(x)\right) \frac{d}{d x} y(x) \\
& +4(g(x)+G) y(x) \frac{d}{d x} g(x) \\
& +(g(x)+G)^{2} \frac{d}{d x} y(x) \\
& y_{n}^{(6)}=\left(\frac{d^{4}}{d x^{4}} g(x)\right) y(x)+4\left(\frac{d^{3}}{d x^{3}} g(x)\right) \frac{d}{d x} y(x) \\
& +7(g(x)+G) y(x) \frac{d^{2}}{d x^{2}} g(x) \\
& +4\left(\frac{d}{d x} g(x)\right)^{2} y(x) \\
& +6(g(x)+G)\left(\frac{d}{d x} y(x)\right) \frac{d}{d x} g(x) \\
& +(g(x)+G)^{3} y(x) \\
& y_{n}^{(7)}=\left(\frac{d^{5}}{d x^{5}} g(x)\right) y(x)+5\left(\frac{d^{4}}{d x^{4}} g(x)\right) \frac{d}{d x} y(x) \\
& +11(g(x)+G) y(x) \frac{d^{3}}{d x^{3}} g(x) \\
& +15\left(\frac{d}{d x} g(x)\right) y(x) \frac{d^{2}}{d x^{2}} g(x) \\
& +13(g(x)+G)\left(\frac{d}{d x} y(x)\right) \frac{d^{2}}{d x^{2}} g(x) \\
& +10\left(\frac{d}{d x} g(x)\right)^{2} \frac{d}{d x} y(x) \\
& +9(g(x)+G)^{2} y(x) \\
& \frac{d}{d x} g(x)+(g(x)+G)^{3} \frac{d}{d x} y(x)
\end{aligned}
$$

$$
\begin{aligned}
y_{n}^{(8)}= & \left(\frac{d^{6}}{d x^{6}} g(x)\right) y(x)+6\left(\frac{d^{5}}{d x^{5}} g(x)\right) \frac{d}{d x} y(x) \\
& +16(g(x)+G) y(x) \frac{d^{4}}{d x^{4}} g(x)+26\left(\frac{d}{d x} g(x)\right) y(x) \\
& \frac{d^{3}}{d x^{3}} g(x)+24(g(x)+G)\left(\frac{d}{d x} y(x)\right) \frac{d^{3}}{d x^{3}} g(x) \\
& +15\left(\frac{d^{2}}{d x^{2}} g(x)\right)^{2} y(x)+48\left(\frac{d}{d x} g(x)\right) \\
& \left(\frac{d}{d x} y(x)\right) \frac{d^{2}}{d x^{2}} g(x)+22(g(x)+G)^{2} y(x) \\
& \frac{d^{2}}{d x^{2}} g(x)+28(g(x)+G) y(x)\left(\frac{d}{d x} g(x)\right)^{2} \\
& +12(g(x)+G)^{2}\left(\frac{d}{d x} y(x)\right) \frac{d}{d x} g(x) \\
& +(g(x)+G)^{4} y(x)
\end{aligned}
$$

-Based on the above formulae and substituting them in the expressions of the Local Truncation Error we can produce formulae of the local errors which are dependent from the energy $E$.

-We study two cases in terms of the value of $E$ within the Local Truncation Error analysis :

1.The Energy is close to the potential, i.e., $G=V_{c}-E \approx 0$. Consequently, the free terms of the polynomials in $G$ are considered only. Thus, for these values of $G$, the methods are of comparable accuracy. This is because the free terms of the polynomials in $G$ are the same for the cases of the classical method and of the methods with vanished the phase-lag and its derivatives.

2. $G>>0$ or $G<<0$. Then $|G|$ is a large number.

-Finally we compute the asymptotic expansions of the Local Truncation Errors

The following asymptotic expansions of the Local Truncation Errors are obtained based on the analysis presented above :

\subsection{Classical Method}

$$
\operatorname{LTE}_{C L}=h^{6}\left(\frac{161}{2400} y(x) G^{3}+\cdots\right)+O\left(h^{8}\right)
$$


4.5 The Method with Vanished Phase-Lag

Produced in [1]

$L T E_{\text {MethAnasSim }}=h^{6}\left(\frac{161}{2400} g(x) y(x) G^{2}+\cdots\right)+O\left(h^{8}\right)$

\subsection{The New Proposed Method with Vanished} Phase-Lag and its First Derivative Produced in Section 3

$$
\begin{aligned}
\text { LTE }_{\text {FourStepII }}= & h^{6}\left[\left(\frac{161}{2400}(g(x))^{2} y(x)\right.\right. \\
& +\frac{161}{1200}\left(\frac{d}{d x} g(x)\right) \frac{d}{d x} y(x)+\frac{161}{480} \\
& \left.\left.\left(\frac{d^{2}}{d x^{2}} g(x)\right) y(x)\right) G+\cdots\right]+O\left(h^{8}\right)(28)
\end{aligned}
$$

From the above equations we have the following theorem:

Theorem 2.For the Classical Four-Step Explicit Method, the error increases as the third power of G. For the Four-Step Explicit Phase-Fitted Method developed in [1] , the error increases as the second power of G. Finally, for the new obtained Four-Step Explicit Method with Vanished Phase-lag and its First Derivative, the error increases as the first power of G. So, for the numerical solution of the time independent radial Schrödinger equation the New Proposed Method with Vanished Phase-Lag and its First Derivative is the most efficient from theoretical point of view, especially for large values of $|G|=\left|V_{c}-E\right|$.

\section{Stability Analysis}

In order to investigate the stability of the new developed methods, we apply them to the scalar test equation:

$$
y^{\prime \prime}=-\omega^{2} y .
$$

This leads to the following difference equation:

$$
\begin{aligned}
A_{2}(s, v)\left(y_{n+2}+y_{n-2}\right) & +A_{1}(s, v)\left(y_{n+1}+y_{n-1}\right) \\
& +A_{0}(s, v) y_{n}=0
\end{aligned}
$$

where

$$
\begin{aligned}
A_{2}(s, v)=1, A_{0}(s, v) & =-\frac{1}{10}+\frac{1}{10} \frac{T_{10}}{\sin (v) v^{3}} \\
A_{0}(s, v) & =-\frac{9}{5}+\frac{1}{5} \frac{T_{11}}{\sin (v) v^{3}}
\end{aligned}
$$

where

$$
\begin{aligned}
T_{10}= & s^{2}(-20 v \sin (2 v)+18-20 \cos (2 v) \\
& +\sin (v) v+2 \cos (v)) \\
T_{11}= & s^{2}(24 \sin (v) v+5 v \sin (3 v) \\
& -\cos (2 v)-8 \cos (v)+10 \cos (3 v)-1) \\
\text { and } s= & \omega h .
\end{aligned}
$$

Remark. The frequency of the scalar test equation (29), $\omega$, is not equal with the frequency of the scalar test equation (4), $\phi$, i.e. $\omega \neq \phi$.

Based on the analysis presented in Section 2, we have the following definitions:

Definition 5.(see [16]) A method is called P-stable if its interval of periodicity is equal to $(0, \infty)$.

Definition 6.A method is called singularly almost $P$-stable if its interval of periodicity is equal to $(0, \infty)-S^{2}$ only when the frequency of the phase fitting is the same as the frequency of the scalar test equation, i.e. $s=v$.

In Figure 3 we present the $s-v$ plane for the method developed in this paper (First Case). In Figure 4 we present the $s-v$ plane for the method developed in this paper (Second Case).

Remark. A shadowed area denotes the $s-v$ region where the method is stable, while a white area denotes the region where the method is unstable.

Remark. There are cases where it is appropriate to observe the surroundings of the first diagonal of the $s-v$ plane. These cases have mathematical models where in order to apply the new produced methods the frequency of the phase fitting must be equal to the frequency of the scalar test equation. The cases are many problems in sciences and engineering (for example the time independent Schrödinger equation).

Based on the above remark, the case where the frequency of the scalar test equation is equal with the frequency of phase fitting is now studied, i.e. we investigate the case where $s=v$ (i.e. see the surroundings of the first diagonal of the $s-v$ plane). Based on this study we extract the results that the interval of periodicity

2 where $S$ is a set of distinct points 


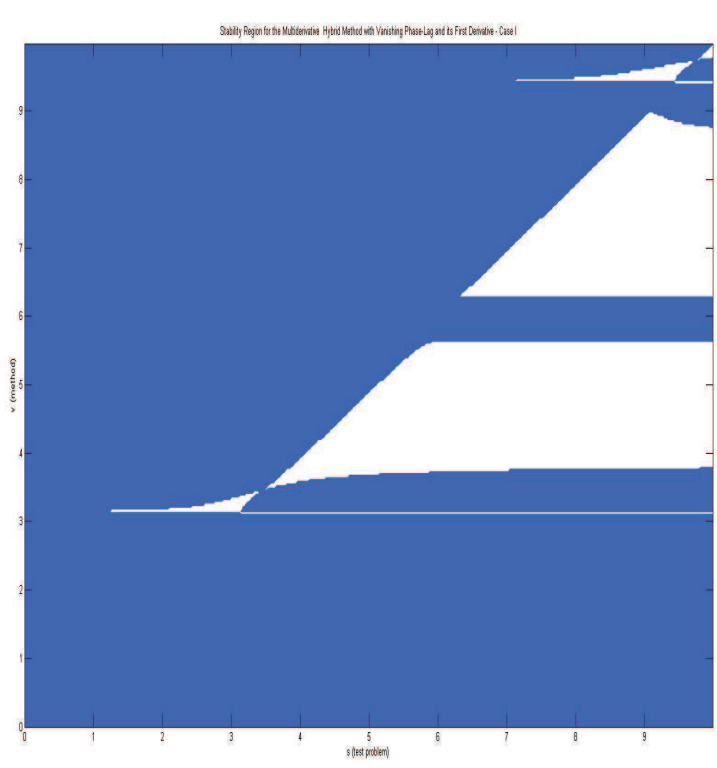

Fig. 3: $s-v$ plane of the the new obtained method with vanished phase-lag and its first derivative (First Case)

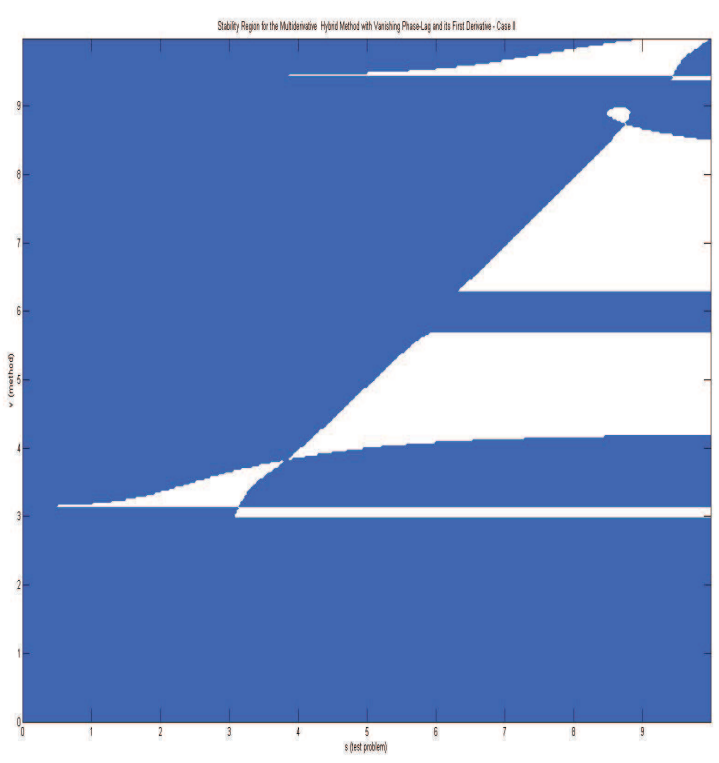

Fig. 4: $s-v$ plane of the the new obtained method with vanished phase-lag and its first derivative (Second Cas)

of the new methods developed in section 3 are equal to: $(0,8.0)$ (First Case) and $(0,9.9)$ (Second Case).
The above investigation leads to the following theorem:

Theorem 3.The methods developed in section 3:

-are of fourth algebraic order,

-have the phase-lag and its first derivative equal to zero -have an interval of periodicity equals to: $(0,8.0)$ (First Case) and $(0,9.9)$ (Second Case) when the frequency of the scalar test equation is equal with the frequency of phase fitting

\section{Numerical results}

The efficiency of the new proposed explicit four-step methods is investigated via the approximate solution of the radial time-independent Schrödinger equation.

The one-dimensional time independent Schrödinger equation with mathematics model given by:

$$
y^{\prime \prime}(r)=\left[l(l+1) / r^{2}+V(r)-k^{2}\right] y(r) .
$$

is a boundary value problem which has the following boundary conditions :

$$
y(0)=0
$$

and another boundary condition, for large values of $r$, determined by physical properties of the specific problem.

We give the following definitions of the functions, quantities and parameters for the above mathematical model (32) :

1.The function $W(r)=l(l+1) / r^{2}+V(r)$ is called the

effective potential. This satisfies $W(x) \rightarrow 0$ as $x \rightarrow \infty$,

2.The quantity $k^{2}$ is a real number denoting the energy,

3.The quantity $l$ is a given integer representing the angular momentum,

4. $V$ is a given function which denotes the potential.

In order the new obtained methods to be applied to any problem, and since these methods are frequency dependent methods, the value of parameter $\phi$ (see for example the notation after (4) and the formulae in section 3 ) must be defined. The parameter $\phi$ for the case of the radial Schrödinger equation is given by (for $l=0$ ) :

$$
\phi=\sqrt{\left|V(r)-k^{2}\right|}=\sqrt{|V(r)-E|}
$$

where $V(r)$ is the potential and $E$ is the energy.

\subsection{Woods-Saxon potential}

For the purpose of our numerical tests we use the well known Woods-Saxon potential. This can be written as :

$$
V(r)=\frac{u_{0}}{1+q}-\frac{u_{0} q}{a(1+q)^{2}}
$$


with $q=\exp \left[\frac{r-X_{0}}{a}\right], u_{0}=-50, a=0.6$, and $X_{0}=7.0$.

The behavior of Woods-Saxon potential is shown in Figure 5.

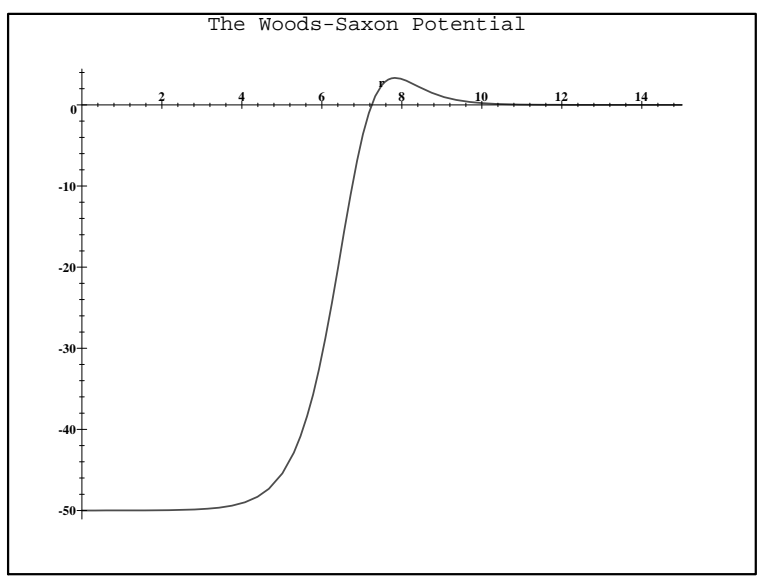

Fig. 5: The Woods-Saxon potential.

For some potentials, such as the Woods-Saxon potential, and using their investigation, some critical points are defined and these points are used in order the parameter $\phi$ to be defined (see for details [36]).

For the purpose of our tests, it is appropriate to choose $\phi$ as follows (see for details [37] and [38]):

$$
\phi=\left\{\begin{array}{l}
\sqrt{-50+E}, \quad \text { for } r \in[0,6.5-2 h] \\
\sqrt{-37.5+E}, \text { for } r=6.5-h \\
\sqrt{-25+E}, \quad \text { for } r=6.5 \\
\sqrt{-12.5+E}, \text { for } r=6.5+h \\
\sqrt{E}, \quad \text { for } r \in[6.5+2 h, 15]
\end{array}\right.
$$

For example, in the point of the integration region $r=$ $6.5-h$, the value of $\phi$ is equal to: $\sqrt{-37.5+E}$. So, $w=$ $\phi h=\sqrt{-37.5+E} h$. In the point of the integration region $r=6.5-3 h$, the value of $\phi$ is equal to: $\sqrt{-50+E}$, etc.

\subsection{Radial Schrödinger Equation - The Resonance Problem}

We will study the numerical solution of the radial time independent Schrödinger equation (32) with the Woods-Saxon potential (35) for the examination of the efficiency of the new proposed methods. The approximation of the true (infinite) interval of integration by a finite one is necessary for the approximate solution of this problem. We take the integration interval $r \in[0,15]$ for the purposes of our numerical experiments.

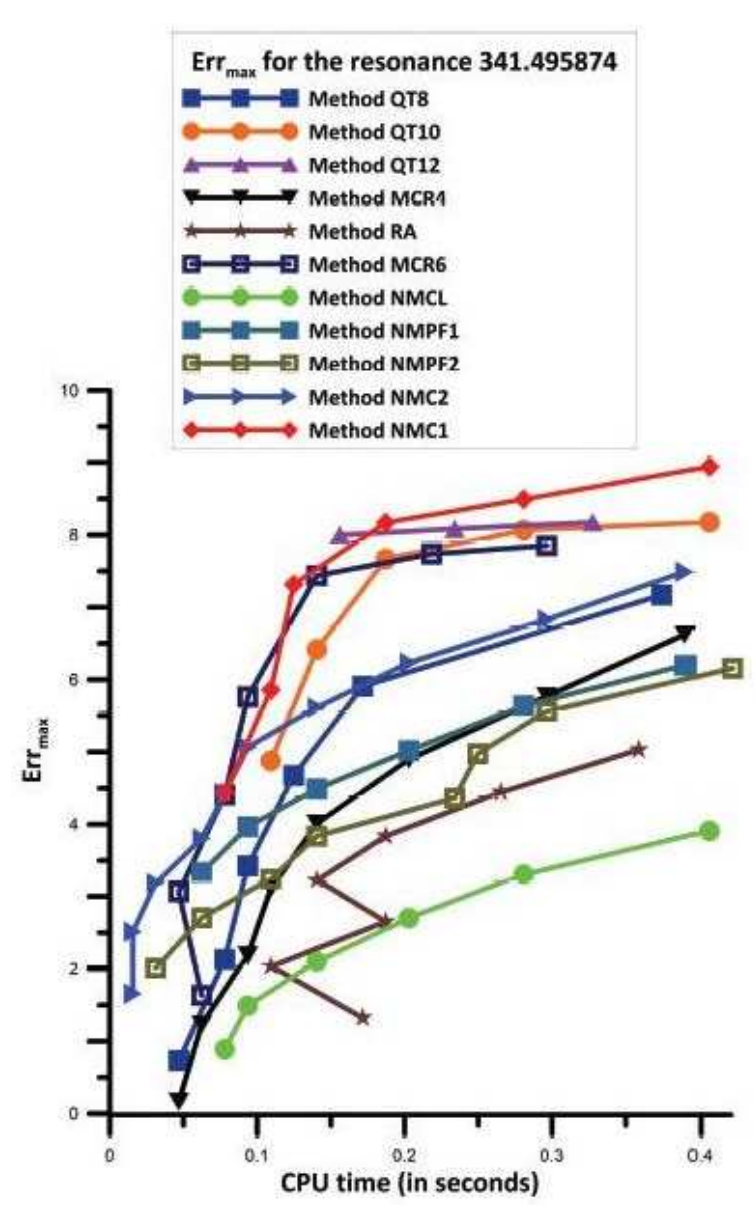

Fig. 6: Accuracy (Digits) for several values of $C P U$ Time (in Seconds) for the eigenvalue $E_{2}=341.495874$. The nonexistence of a value of Accuracy (Digits) indicates that for this value of CPU, Accuracy (Digits) is less than 0

We consider equation (32) in a rather large domain of energies, i.e., $E \in[1,1000]$.

In the case of positive energies, $E=k^{2}$, the potential decays faster than the term $\frac{l(l+1)}{r^{2}}$ and the Schrödinger equation effectively reduces to

$$
y^{\prime \prime}(r)+\left(k^{2}-\frac{l(l+1)}{r^{2}}\right) y(r)=0
$$

for $r$ greater than some value $R$.

The above equation has linearly independent solutions $k r j_{l}(k r)$ and $k r n_{l}(k r)$, where $j_{l}(k r)$ and $n_{l}(k r)$ are the spherical Bessel and Neumann functions respectively. Thus, the solution of equation (32) (when $r \rightarrow \infty$ ), has the asymptotic form

$$
\begin{array}{r}
y(r) \approx A k r j_{l}(k r)-B k r n_{l}(k r) \\
\approx A C\left[\sin \left(k r-\frac{l \pi}{2}\right)+\tan d_{l} \cos \left(k r-\frac{l \pi}{2}\right)\right]
\end{array}
$$


where $\delta_{l}$ is the phase shift that may be calculated from the formula

$$
\tan \delta_{l}=\frac{y\left(r_{2}\right) S\left(r_{1}\right)-y\left(r_{1}\right) S\left(r_{2}\right)}{y\left(r_{1}\right) C\left(r_{1}\right)-y\left(r_{2}\right) C\left(r_{2}\right)}
$$

for $r_{1}$ and $r_{2}$ distinct points in the asymptotic region (we choose $r_{1}$ as the right hand end point of the interval of integration and $\left.r_{2}=r_{1}-h\right)$ with $S(r)=k r j_{l}(k r)$ and $C(r)=-k r n_{l}(k r)$. Since the problem is treated as an initial-value problem, we need $y_{j}, j=0,(1) 3$ before starting a four-step method. From the initial condition, we obtain $y_{0}$. The values $y_{i}, i=1(1) 3$ are obtained by using high order Runge-Kutta-Nyström methods(see [39] and [40]). With these starting values, we evaluate at $r_{2}$ of the asymptotic region the phase shift $\delta_{l}$.

For positive energies, we have the so-called resonance problem. This problem consists either of finding the phase-shift $\delta_{l}$ or finding those $E$, for $E \in[1,1000]$, at which $\delta_{l}=\frac{\pi}{2}$. We actually solve the latter problem, known as the resonance problem.

The boundary conditions for this problem are:

$$
y(0)=0, y(r)=\cos (\sqrt{E} r) \text { for large } r .
$$

We compute the approximate positive eigenenergies of the Woods-Saxon resonance problem using:

-The eighth order multi-step method developed by Quinlan and Tremaine [41], which is indicated as Method QT8.

-The tenth order multi-step method developed by Quinlan and Tremaine [41], which is indicated as

Method QT10.

-The twelfth order multi-step method developed by Quinlan and Tremaine [41], which is indicated as Method QT12.

-The fourth algebraic order method of Chawla and Rao with minimal phase-lag [43], which is indicated as Method MCR4

-The exponentially-fitted method of Raptis and Allison [42], which is indicated as Method RA

-The hybrid sixth algebraic order method developed by Chawla and Rao with minimal phase-lag [44], which is indicated as Method MCR6

-The classical form of the fourth algebraic order fourstep method developed in Section 3, which is indicated as Method NMCL ${ }^{3}$.

-The Phase-Fitted Method (Case 1) developed in [1], which is indicated as Method NMPF1

-The Phase-Fitted Method (Case 2) developed in [1], which is indicated as Method NMPF2

-The New Obtained Method developed in Section 3 (Case 2), which is indicated as Method NMC2

-The New Obtained Method developed in Section 3 (Case 1), which is indicated as Method NMC1

\footnotetext{
3 with the term classical we mean the method of Section 3 with constant coefficients
}

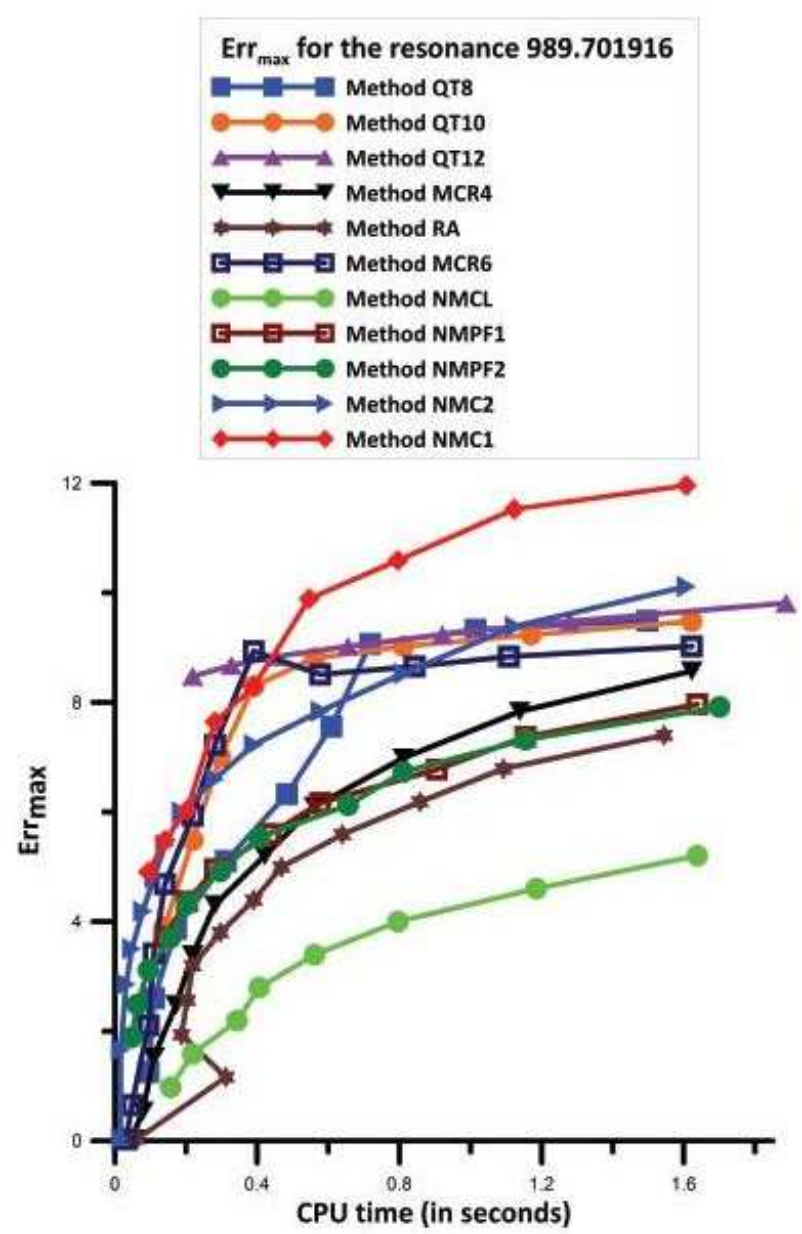

Fig. 7: Accuracy (Digits) for several values of $C P U$ Time (in Seconds) for the eigenvalue $E_{2}=341.495874$. The nonexistence of a value of Accuracy (Digits) indicates that for this value of CPU, Accuracy (Digits) is less than 0

The numerically calculated eigenenergies are compared with reference values ${ }^{4}$. In Figures 8 and 9, we present the maximum absolute error $E r r_{\text {max }}=\left|\log _{10}(E r r)\right|$ where

$$
E r r=\left|E_{\text {calculated }}-E_{\text {accurate }}\right|
$$

of the eigenenergies $E_{2}=341.495874$ and $E_{3}=989.701916$ respectively, for several values of CPU time (in seconds). We note that the CPU time (in seconds) counts the computational cost for each method.

\footnotetext{
4 the reference values are computed using the well known twostep method of Chawla and Rao [44] with small step size for the integration
} 


\section{Conclusions}

In this paper, we studied a family of explicit four-step methods first introduced by Anastassi and Simos [1]. The main purpose of this investigation is the study of the elimination of the phase-lag and its first derivative of the above mentioned family of the methods. For this family we presented a comparative error and stability analysis. We have also investigated the effect of the vanishing of the phase-lag and its first derivative on the efficiency of the above mentioned methods for the approximate solution of the radial Schrödinger equation and related problems.

From the results presented above, we can make the following remarks:

1.The classical form of the tenth algebraic order four-step multiderivative method developed in Section 3, which is indicated as Method NMCL is more efficient than the fourth algebraic order method of Chawla and Rao with minimal phase-lag [43], which is indicated as Method MCR4. Both the above mentioned methods are more efficient than the exponentially-fitted method of Raptis and Allison [42], which is indicated as Method RA.

2.The tenth algebraic order multistep method developed by Quinlan and Tremaine [41], which is indicated as Method QT10 is more efficient than the fourth algebraic order method of Chawla and Rao with minimal phase-lag [43], which is indicated as Method MCR4. The Method QT10 is also more efficient than the eighth order multi-step method developed by Quinlan and Tremaine [41], which is indicated as Method QT8. Finally, the Method QT10 is more efficient than the hybrid sixth algebraic order method developed by Chawla and Rao with minimal phase-lag [44], which is indicated as Method MCR6 for large CPU time and less efficient than the Method MCR6 for small CPU time.

3.The twelfth algebraic order multistep method developed by Quinlan and Tremaine [41], which is indicated as Method QT12 is more efficient than the tenth order multistep method developed by Quinlan and Tremaine [41], which is indicated as Method QT10

4.The Phase-Fitted Method (Case 1) developed in [1], which is indicated as Method NMPF1 is more efficient than the classical form of the fourth algebraic order four-step method developed in Section 3, which is indicated as Method NMCL, the exponentially-fitted method of Raptis and Allison [42] and the Phase-Fitted Method (Case 2) developed in [1], which is indicated as Method NMPF2

5.The New Obtained Method developed in Section 3 (Case 2), which is indicated as Method NMC2 is more efficient than the classical form of the fourth algebraic order four-step method developed in Section 3, which is indicated as Method NMCL, the exponentially-fitted method of Raptis and Allison [42] and the Phase-Fitted Method (Case 2) developed in [1], which is indicated as Method NMPF2 and the Phase-Fitted Method (Case 1) developed in [1], which is indicated as Method NMPF1

6.The New Obtained Method developed in Section 3 (Case 2), which is indicated as Method NMC1 is the most efficient one.

All computations were carried out on a IBM PC-AT compatible 80486 using double precision arithmetic with 16 significant digits accuracy (IEEE standard).

\section{References}

[1] Z. A. Anastassi and T. E. Simos, A parametric symmetric linear four-step method for the efficient integration of the Schrödinger equation and related oscillatory problems, JOURNAL OF COMPUTATIONAL AND APPLIED MATHEMATICS, 236, 3880-3889 (2012).

[2] A. D. Raptis and T. E. Simos: A four-step phase-fitted method for the numerical integration of second order initialvalue problem, BIT, 31, 160-168 (1991).

[3] D. G. Quinlan and S. Tremaine, Symmetric Multistep Methods for the Numerical Integration of Planetary Orbits, The Astronomical Journal, 100, 1694-1700 (1990).

[4] J. M. Franco, M. Palacios, JOURNAL OF COMPUTATIONAL AND APPLIED MATHEMATICS, 30, 1 (1990).

[5] J. D.Lambert, Numerical Methods for Ordinary Differential Systems, The Initial Value Problem, John Wiley and Sons, 104-107 (1991).

[6] E. Stiefel, D. G. Bettis, Stabilization of Cowell's method, Numer. Math., 13, 154-175 (1969).

[7] G. A. Panopoulos, Z. A. Anastassi and T.E. Simos: Two New Optimized Eight-Step Symmetric Methods for the Efficient Solution of the Schrödinger Equation and Related Problems, MATCH Commun. Math. Comput. Chem., 60, 773-785 (2008).

[8] G. A. Panopoulos, Z. A. Anastassi and T. E. Simos, Two optimized symmetric eight-step implicit methods for initial-value problems with oscillating solutions, Journal of Mathematical Chemistry, 46, 604-620 (2009).

[9] http://www.burtleburtle.net/bob/math/multistep.html.

[10] T. E. Simos and P. S. Williams, Bessel and Neumann fitted methods for the numerical solution of the radial Schrödinger equation, Computers and Chemistry, 21, 175-179 (1977).

[11] T. E. Simos and Jesus Vigo-Aguiar, A dissipative exponentially-fitted method for the numerical solution of the Schrödinger equation and related problems, Computer Physics Communications, 152, 274-294 (2003).

[12] T. E. Simos and G. Psihoyios, JOURNAL OF COMPUTATIONAL AND APPLIED MATHEMATICS, 175, IX-IX (2005).

[13] T. Lyche, Chebyshevian multistep methods for Ordinary Differential Eqations, Num. Math., 19, 65-75 (1972).

[14] T. E. Simos and P. S. Williams, A finite-difference method for the numerical solution of the Schrdinger equation, Journal of Computational and Applied Mathematics, 79, 189-205 (1997). 
[15] R. M. Thomas, Phase properties of high order almost Pstable formulae, BIT, 24, 225-238 (1984).

[16] J. D. Lambert and I. A. Watson, Symmetric multistep methods for periodic initial values problems, J. Inst. Math. Appl., 18, 189-202 (1976).

[17] A. Konguetsof and T. E. Simos, A generator of hybrid symmetric four-step methods for the numerical solution of the Schrödinger equation, JOURNAL OF COMPUTATIONAL AND APPLIED MATHEMATICS, 158, 93-106 (2003).

[18] Z. Kalogiratou, T. Monovasilis and T.E. Simos, Symplectic integrators for the numerical solution of the Schrödinger equation, JOURNAL OF COMPUTATIONAL AND APPLIED MATHEMATICS, 158, 83-92 (2003).

[19] Z. Kalogiratou and T. E. Simos, Newton-Cotes formulae for long-time integration, JOURNAL OF COMPUTATIONAL AND APPLIED MATHEMATICS, 158, 75-82 (2003).

[20] G. Psihoyios and T. E. Simos, Trigonometrically fitted predictor-corrector methods for IVPs with oscillating solutions, JOURNAL OF COMPUTATIONAL AND APPLIED MATHEMATICS, 158, 135-144 (2003).

[21] T. E. Simos, I. T. Famelis and C. Tsitouras, Zero dissipative, explicit Numerov-type methods for second order IVPs with oscillating solutions, NUMERICAL ALGORITHMS, 34, 27-40 (2003).

[22] T. E. Simos, Dissipative trigonometrically-fitted methods for linear second-order IVPs with oscillating solution, APPLIED MATHEMATICS LETTERS, 17, 601-607 (2004).

[23] K. Tselios and T.E. Simos, Runge-Kutta methods with minimal dispersion and dissipation for problems arising from computational acoustics, JOURNAL OF COMPUTATIONAL AND APPLIED MATHEMATICS, 175, 173-181 (2005).

[24] D. P. Sakas and T. E. Simos, Multiderivative methods of eighth algrebraic order with minimal phase-lag for the numerical solution of the radial Schrödinger equation, JOURNAL OF COMPUTATIONAL AND APPLIED MATHEMATICS, 175, 161-172(2005).

[25] G. Psihoyios and T. E. Simos, A fourth algebraic order trigonometrically fitted predictor-corrector scheme for IVPs with oscillating solutions, JOURNAL OF COMPUTATIONAL AND APPLIED MATHEMATICS, 175, 137-147 (2005).

[26] Z. A. Anastassi and T. E. Simos, An optimized Runge-Kutta method for the solution of orbital problems, JOURNAL OF COMPUTATIONAL AND APPLIED MATHEMATICS, 175, 1-9 (2005).

[27] T. E. Simos, Closed Newton-Cotes trigonometrically-fitted formulae of high order for long-time integration of orbital problems, APPLIED MATHEMATICS LETTERS, 22, 1616-1621 (2009).

[28] S. Stavroyiannis and T. E. Simos, Optimization as a function of the phase-lag order of nonlinear explicit two-step P-stable method for linear periodic IVPs, APPLIED NUMERICAL MATHEMATICS, 59, 2467-2474 (2009).

[29] T. E. Simos, Exponentially and Trigonometrically Fitted Methods for the Solution of the Schrödinger Equation, ACTA APPLICANDAE MATHEMATICAE, 110, 13311352 (2010).
[30] T. E. Simos, New Stable Closed Newton-Cotes Trigonometrically Fitted Formulae for Long-Time Integration, Abstract and Applied Analysis, 2012, 15 pages (2012).

[31] T. E. Simos, Optimizing a Hybrid Two-Step Method for the Numerical Solution of the Schrödinger Equation and Related Problems with Respect to Phase-Lag, Journal of Applied Mathematics, 2012, 17 pages (2012).

[32] Z. A. Anastassi and T. E. Simos, A parametric symmetric linear four-step method for the efficient integration of the Schrödinger equation and related oscillatory problems, JOURNAL OF COMPUTATIONAL AND APPLIED MATHEMATICS, 236, 3880-3889 (2012).

[33] Bo Zhang and Zhicai Juan, Modeling User Equilibrium and the Day-to-day Traffic Evolution based on Cumulative Prospect Theory, Information Science Letters, 1, 9-12 (2013).

[34] D. Kundu, A. Sarhan and Rameshwar D. Gupta, On SarhanBalakrishnan Bivariate Distribution, Journal of Statistics Applications \& Probability, 1, 163-170 (2012).

[35] G. A. Panopoulos and T.E. Simos, An Optimized Symmetric 8-Step Semi-Embedded Predictor-Corrector Method for IVPs with Oscillating Solutions, Applied Mathematics \& Information Sciences, 7, 73-80 (2013).

[36] L. Gr. Ixaru and M. Rizea, Comparison of some fourstep methods for the numerical solution of the Schrödinger equation, Comput. Phys. Commun., 38, 329-337 (1985).

[37] L. Gr. Ixaru and M. Micu, Topics in Theoretical Physics, Central Institute of Physics, Bucharest, (1978).

[38] L. Gr. Ixaru and M. Rizea, A Numerov-like scheme for the numerical solution of the Schrödinger equation in the deep continuum spectrum of energies, Computer Physics Communications, 19, 23-27 (1980).

[39] J. R. Dormand, M. E. A. El-Mikkawy and P. J. Prince, Families of Runge-Kutta-Nyström formulae, IMA J. Numer. Anal., 7, 235-250 (1987).

[40] J. R. Dormand and P. J. Prince, A family of embedded RungeKutta formulae, J. Comput. Appl. Math., 6, 19-26 (1980).

[41] G. D. Quinlan and S. Tremaine, Symmetric Multistep Methods for the Numerical Integration of Planetary Orbits, The Astronomical Journal, 100, 1694-1700 (1990).

[42] A. D. Raptis and A. C. Allison, Exponential-fitting methods for the numerical solution of the Schrödinger equation, Computer Physics Communications, 14, 1-5 ( 1978).

[43] M. M. Chawla and P. S. Rao, An Noumerov-typ method with minimal phase-lag for the integration of second order periodic initial-value problems II Explicit Method, Journal of Computational and Applied Mathematics, 15, 329-337 (1986).

[44] M. M. Chawla and P. S. Rao, An explicit sixth - order method with phase-lag of order eight for $y^{\prime \prime}=f(t, y)$, Journal of Computational and Applied Mathematics, 17, 363-368 (1987). 


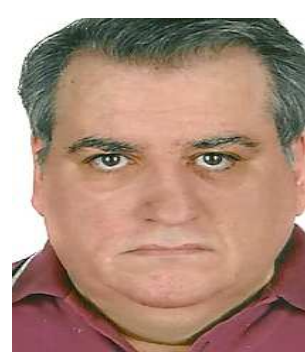

Theodore E. Simos

(b. 1962 in Athens, Greece).

He holds a Ph.D. on

Numerical Analysis (1990)

from the Department

of Mathematics

of the National Technical

University of Athens,

Greece. He is Highly Cited

Researcher in Mathematics

(http://isihighlycited.com/), Active Member of the European Academy of Sciences and Arts, Active Member of the European Academy of Sciences and Corresponding Member of European Academy of Sciences, Arts and Letters. He is Senior Editor of the Journal: Applied Mathematics and Computation (Elsevier, INC), Editor-in-Chief of three scientific journals and editor of more than 25 scientific journals. He is reviewer in several other scientific journals and conferences. His research interests are in numerical analysis and specifically in numerical solution of differential equations, scientific computing and optimization. He is the author of over 400 peer-reviewed publications and he has more than 2000 citations (excluding self-citations). 